\title{
Evidence-Based Pharmacotherapy of Epilepsy
}

\author{
Zahra Tolou Ghamari $^{1{ }^{1}}$; Jafar Mehavari Habibabadi $^{2}$; Abbas Ali Palizban ${ }^{3}$

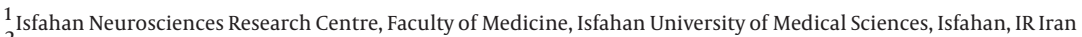 \\ 2 Department of Neurology, Faculty of Medicine, Isfahan University of Medical Sciences, Isfahan, IR Iran \\ 3 Department of Clinical Biochemistry, Isfahan University of Medical Sciences, Isfahan, IR Iran \\ ${ }^{*}$ Corresponding author: Zahra Tolou Ghamari, Department of Neurology, Isfahan Neurosciences Research Centre, Isfahan University of Medical Sciences, Isfahan, IR Iran. Tel: +98- \\ 3136291050, Fax: +98-3136680011, E-mail: toloeghamari@pharm.mui.ac.ir \\ Received: February 24, 2014; Revised: Mar 3, 2014; Accepted: Mar 11, 2014
}

\begin{abstract}
Background: Due to the narrow therapeutic window of the AEDs prescription, their side effects and efficacy are the most important items that should be considered in epileptic patients.

Objectives: This study aimed to investigate evidence-based pharmacotherapy in epileptic patients.

Patients and Methods: Data were collected Cross-sectionally from patients $(n=24)$ registered at Kashani Epilepsy Ward in Isfahan. Demographic, clinical, hematology and biochemical data were recorded in d-base and analyzed using SPSS application for windows. Results: The frequency of polypharmacy was 79\% in which 50\% of epileptic patients received 3 to 4 AEDs. The onset of seizure was under 16 years old in 70\% of patients. Fourteen varieties of AEDs were used, among them valproic acid (Depakote) was the most administered drugs. Prescriptions of two patients consisted of 4 and 6 AEDs: patient with code No. 575 (carbamazepine, topiramate, clobasam, lamotrigine) and another one with code No. 587 (oxcarbamazepine, phenytoin, gabapentin, valproic acid, clonazepam, lamotrigine). Red blood cell counts, hemoglobin and hematocrit in patients under more than one drug treatment were significantly lower than patients with AED monotherapy.

Conclusions:AEDs are well-recognized to control seizure attacks. In clinical practice, the older generation of AEDs such as carbamazepine (CBZ), valporic acid (VPA), phenytoin (PHT), topiramate (TOP) and lamotrigine (LAMO) might need monitoring serum levels. Nonrational polypharmacy in terms of simultaneous using of both AEDs inducer and inhibitor could cause sedation, dizziness, and cognitive adverse effects. Further studies are needed to confirm these associations. Finally, to avoid polypharmacy that could arise side effects, a sufficient intervention for each AED to decide on its continuation, interruption or the number of drugs should be attempted. Combinations based on $\mathrm{CBZ}+\mathrm{VP}, \mathrm{VP}+\mathrm{TOP}, \mathrm{CBZ}+\mathrm{TOP}, \mathrm{VP}+\mathrm{LAMO}$, and TOP + LAMO could cause pharmacokinetic interactions.
\end{abstract}

Keywords:Epilepsy; Pharmacotherpay; AEDs; Evidence-Based

\section{Background}

To achieve a suitable pharmacotherapy strategy in epileptic patients, evidence-based study regarding the drug prescription seems to be important. The consequences of case classification and judgment are not only difficult for clinical supervision, but also need to consider plenty of vigilance on patients' care.

The clinical case definition of antiepileptic drugs (AEDs), must comprise such factors as interference of each drug, their significant results, monitoring signs of achievement or breakdown, number of the drugs, which should be tried, accounting seizure incidence, and the role of adverse effects and acceptability.

Alternatively, the philosophy of evidence-based pharmacotherapy (EBP) is to provide a methodical advance in integrating the best obtainable evidence into the procedure of quantifiable judgments for individual patients (1-6). The prescription of AEDs is supported by the recog- nition of their efficiency and side effects. Because of the comparable efficacies between AEDs, patient's management is distinguished by side effects. Dissimilarities in categorization of side effects, and inconsistency within epileptic population make it difficult to use information on side effects and decide on suitable AEDs.

Decreasing glutamate excitation (Glu) and increasing $\gamma$-aminobutyric acid (GABA) inhibition are parts of the mechanisms of action for AEDs. Ionic channels could be potentially accountable for their action too. Carbamazepine, phenytoin, primidone, oxcarbazepine, and possibly sodium valproate affect sodium channels and potentiate GABA receptors. Valproic acid and gabapentin increase the formation of GABA. Gabapentin action may be associated with an active carrier system in the neurons (7-16).

Lamotrigine affects sodium-channels by extending inactivation and though it is less capable in maintaining ace-

Implication for health policy/practice/research/medical education:

A practical approach to antiepileptic drugs (AEDs) prescription should address the obtained information from Iranian clinical AEDs assessments, as well as individual characteristics of the patients and complicated aspects like bioavailability (pharmacokinetics) parameters. Therefore, predicting the hidden drug-drug interactions by studying the individualized approaches for most patients seem to be useful for getting an effectual and well-tolerated AEDs-regimen.

Copyright (C 2015, Tehran University of Medical Sciences. This is an open-access article distributed under the terms of the Creative Commons Attribution License, which permits unrestricted use, distribution, and reproduction in any medium, provided the original work is properly cited. 
tylcholine or GABA, it prevents the release of glutamate and aspartate $(7,17)$. Topiramate releases antagonism of the excitatory receptor (Glu), blocks the action potentials in a time-dependent behavior, hence, increases the action of the GABA and chloride channels $(7,18)$.

Clonazepam supports GABAergic in the brain $(7,19)$. Levetiracetam blocks nerve transmission across synapses by linking to vesicle synaptic protein $(7,20)$. The mechanism of action for clobazam is associated with GABA receptor $(7,21)$. Phenobarbital potentiates the effect of GABA at receptor site and also blocks a subtype of glutamate receptor 0020 (7, 22). Zonisamide enhances GABA discharge, inhibits Glu release, voltage-gated sodium channels, and T-type calcium channels $(7,23)$.

Common adverse reactions of AEDs are vertigo, sleepiness, cerebral deliberating and hematologic adverse effects such as purpura, anemia, thrombocytopenia, lymphadenopathy, increased white blood cell count, lymphocytosis, non-Hodgkin's lymphoma, and increased bleeding time. Other side effects may include agranulocytosis, aplastic anemia, leucopenia, and thrombocytopenia $(9,24-26)$.

\section{Objectives}

Administration of AEDs among Iranian epileptic population maintains to increase. In order to achieve drug management, systematic studies on evidence-based pharmacotherapy is important.

\section{Patients and Methods}

A cross-sectional study was carried out on 24 patients (11 females and 13 males) with a mean age of 27 years (ranged, 7-74 years), registered at the Isfahan Kashani Epilepsy Ward affiliated to Isfahan Neurosciences Research Centre (INRCC). There was no induction in treatment procedure. The study was approved by the Institute Research Ethics Committee (IREC). According to AEDs monotherapy or polypharmacy, two groups were made. Group one included patients under one AED (monotherapy) therapy, and the second group consisted of patients treated with more than one AED (polypharmacy). Sex, age, time of seizure onset, biochemical and hematological parameters, types of AEDs, and number of drugs used by each patient were recorded in a d-base. Descriptive statistics such as mean, minimum, and maximum was calculated for variables of interest using SPSS application for windows.

\section{Results}

The number of AEDs used by each patient ranged from 1 to 6 with a mean of 3. Within the studied population, the incidence of polypharmacy was 79\% (Figure 1). Figure 2 shows that the prescription of AEDs in 50\% of patients consisted of 3 to 4 drugs.

In $70 \%$ of the studied population, the time of seizure onset was under 16 years old. Around 14 varieties of AEDs included both generic and trade names that were used.
Valproic acid (Depakote), carbamazepine (Tegretol), phenytoin, lamotrigine, topiramate, clonazepam, levetiracetam, clobasam, primidone, zonisamide, phenobarbital, ethosuximide, gabapentin, oxcarbazepine were the bestselling drugs. Table 1 shows evidence-based pharmacotherapy in a number of studied epileptic patients.

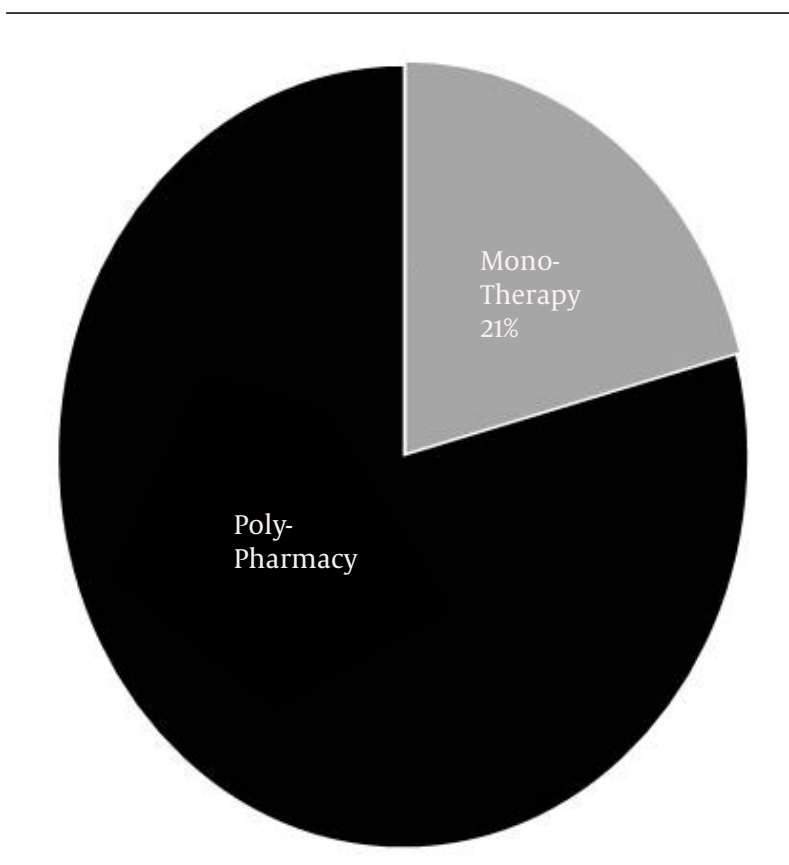

Figure 1. The Percentage of Monotherapy (Group 1) or Polypharmacy (Group 2) in Prescriptions $(\mathrm{n}=24)$

Figure 2. Demonstration of the Number of AEDs Used by Each Patient

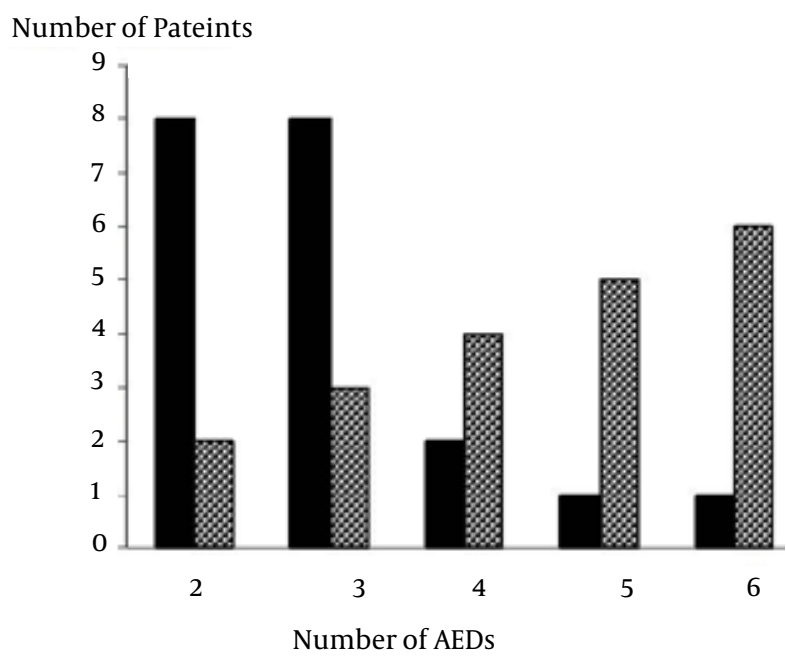

The mean onset of the first epileptic attack was at 11.6 years old, ranged from the beginning of the life to 74 years old. Figure 3 shows the distribution of the onset of first seizure attack. 
Table 1. Evidence-Based Pharmacotherapy in a Number of Studied Epileptic Patients ${ }^{\mathrm{a}}$

562; VP 563, 567, 569; CBZ 576; PHT, Treatment Follows Gold Standard Monotherapy

\begin{tabular}{|c|c|c|}
\hline Patient's code No. & $\begin{array}{l}\text { Type of AEDs administered } \\
\text { for Each patient, varieties of } \\
\text { AEDs in Each Prescription }\end{array}$ & Predicting AEDs Mechanism of Action in Combination Therapy (1-48) \\
\hline 554 & CBZ, PID + VP, PIH + Zoni & $\begin{array}{l}\text { CBZ; Decrease firing in sodium channel, potentate, GABA receptor, VP; possibly } \\
\text { affect sodium channel, increases the formation of GABA, Zoni; inhibits Glu } \\
\text { release, inhibits voltage-gated Na channel, inhibits T-type calcium channel }\end{array}$ \\
\hline 556 & $\mathrm{CBZ}+\mathrm{VP}+\mathrm{LAMO}$ & $\begin{array}{l}\text { LAMO; Extended inactivation affects sodium channels, is less capable in pre- } \\
\text { serving acetylcholine }\end{array}$ \\
\hline 557 & $\mathrm{TOP}+\mathrm{VP}+\mathrm{PHENO}$ & $\begin{array}{l}\text { TOP; blockade the action potential, GABA action } \uparrow \text {, controls chloride channel, } \\
\text { release antagonism of the glutamate excitatory amino acid receptor, PHENO; } \\
\text { GABA receptor affinity, a subtype of glutamate receptor blockage }\end{array}$ \\
\hline 559 & PHT/LEV & $\begin{array}{l}\text { PHT; sodium channel by decreasing firing, potentiate GABA receptor, LEV: } \\
\text { blocks nerve transmission }\end{array}$ \\
\hline 564 & CBZ/PHT/GBP/CLON/LAMO & CLON: support GABA-ergic situated in the brain \\
\hline
\end{tabular}

inductor; PIH, potent inhibitor; TOP, topiramate; VP, valproic acid;ZONI, zonisamide.

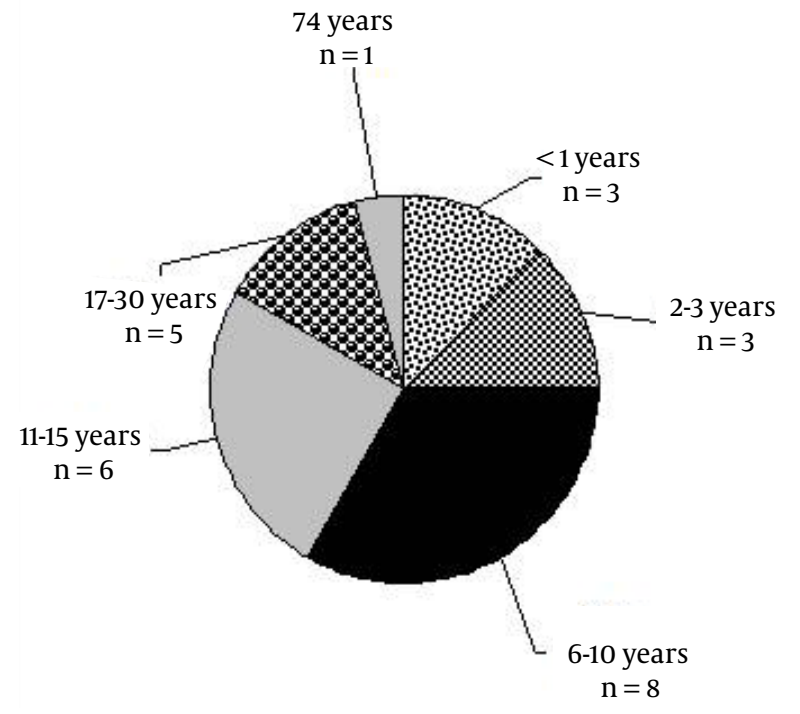

Figure 3. Distribution of the Age of Seizure Onset $(n=24)$

Patients with code numbers 562 (on Depakote), 563, 567, 569 (on carbamazepine), and 576 (on phenytoin) received gold standard monotherapy. Prescriptions were based on two AEDs in patients with code numbers 559 (phenytoin, levetiracetam), 565 (carbamazepinephenytoin compound), 566 (Depakote, lamotrigine), 570 (carbamazepine, valproic acid), 580 (primidone, valproic acid), 582 (carbamazepine, lamotrigine), and 584 (Tegretol, Depakote). Prescriptions were based on three AEDs in patients with code numbers 554 (carbamazepine and Tegretol, valproic acid, zonisamide), 556 (Tegretol, Depakote, lamotrigine), 557 (topiramate, val- proic acid, phenobarbital), 568 (carbamazepine, valproic acid, zonisamide), 571 (carbamazepine, valproic acid, lamotrigine), 583 (primidone, phenytoin, topiramate), 585 (Tegretol, topiramate, Depakote) and 586 (valproic acid, ethosuximide, phenobarbital). In two patients, prescriptions were based on 4 and 6 AEDs: patient with code No. 575 (carbamazepine, topiramate, clobasam, lamotrigine) and in another one with code No. 587 (oxcarbamazepine, phenytoin, gabapentin, valproic acid, clonazepam, lamotrigine).

Table 2 shows the mean, minimum and maximum values of biochemistry and hematology parameters within two groups of epileptic patients. With a high mean value of white blood cell count, the maximum values in both groups (9300 monotherapy; 10200 polypharmacy) were high. Red blood cell count, hemoglobin and hematocrit in patients with more than one AED were significantly lower than patients under monotherapy. With a mean value of 43.2 and a maximum value of 84 , lymphocyte was significantly higher in group two. With a mean value of 252.3, the maximum value in group two was 559 . Blood sugar, with a range of 60-112 mg/dL, does not seem to be significantly different in both groups.

\section{Discussion}

Controlled monotherapy could be used as the usual pharmacotherapy strategy for most of the epileptic cases, but interindividual and intraindividual susceptibility appear to be more significant than the number, form the perspective of AEDs. According to previous researches, alternative monotherapy or early treatment with another AED could result in similar efficacy. In this regard, side effects might be associated with monotherapy or combined therapies (29). 
Table 2. Comparison of Mean, Minimum and Maximum Values Related to Biochemistry and Hematology Results Within Two Groups (Monotherapy and Polypharmacy)

\begin{tabular}{|c|c|c|c|}
\hline \multirow{2}{*}{$\begin{array}{c}\text { Serum Biochemical Parameters, Normal Ranges } \\
\text { White blood cell, } 4400-11000 . \mathrm{mm}^{3}\end{array}$} & \multicolumn{3}{|c|}{ Group 1 vs. Group 2 (Mean, Min, Max) } \\
\hline & 6420 vs. 5568 & 3800 vs. 3200 & 9300 vs. 10200 \\
\hline Red blood cell, M: 4.5-5.9 m/mm³, F: 4.1-5.1 m/mm $/ \mathrm{mm}^{3}$ & 5.24 vs. 4.50 & 4.13 vs. 3.64 & 6.72 vs. 5.76 \\
\hline Hemoglobin, M:14-17.5 g/dL, F: 12.3-15.3 g/dL & 13.22 vs. 12.45 & 11.4 vs. 10.4 & 15.1 vs. 14.3 \\
\hline Hematocrit, M: 41.5-50.5\%, F: 35.9-44.9\% & 40.88 vs. 37.9 & 35.1 vs. 33.8 & 44.9 vs. 43.2 \\
\hline Platelets, $150000-450000 / \mathrm{mm}^{3}$ & 211333 vs. 212812 & 174000 vs. 112000 & 238000 vs. 330000 \\
\hline Lymphocyte, 20-40,\% & 32.9 vs. 43.2 & 24.8 vs. 26.2 & 37.4 vs. 84 \\
\hline Sodium, 135-145, meq/L & 140.2 vs. 139.7 & 136 vs. 135 & 143 vs. 144 \\
\hline Potassium, 3.5-5, meq/L & 4.2 vs. 4.01 & 4 vs. 3.5 & 4.3 vs. 4.6 \\
\hline Blood urea nitrogen, 8-24 mg/dL & 14.2 vs. 12.42 & 11 vs. 9 & 18 vs. 17 \\
\hline Calcium, 8.2-10.6, mg/dL & 8.5 vs. 8.7 & 8.2 vs. 8.0 & 8.9 vs. 9.3 \\
\hline Phosphorous, 2.5-4.5, mg/dL & 4.7 vs. 4.07 & 3.6 vs. 2.5 & 6.7 vs. 5.4 \\
\hline Blood sugar, 70-110, mg/dL & 84.4 vs. 89.1 & 70 vs. 60 & 110 vs. 112 \\
\hline Alkaline phosphates, 60-360 u/L & 163.6 vs. 252.3 & 2.4 vs. 87 & 328 vs. 559 \\
\hline Aspartate aminotransferase, $4-40 \mathrm{u} / \mathrm{L}$ & 25.3 vs. 19.2 & 10 vs. 12 & 39 vs. 42 \\
\hline
\end{tabular}

While the incidence of monotherapy within the studied population in this article was around $21 \%$, there is sufficient data that AEDs side effects in both forms (monotherapy or polytherapy) could influence negatively patients' quality or quantity of life. Many previous studies reported that up to $50 \%$ of patients with epilepsy were treated at least intermittently with more than one AED. In these cases, identification of a specific genetic cause could have a significant personal value, even in the absence of clear clinical utility (30-32). Assortment of AED to control epileptic attack should be based on several factors, including mechanism of action, side effect profile, and AED-AED interactions (7, 33). For example, co-prescription of valproic acid with lamotrigine considerably increases severe cutaneous reactions (34).

Treatment of epileptic attack based on monotherapy or rational polypharmacy is not always contradictory, as many anticonvulsants have different mechanisms of action and combination therapy may potentiate their effects $(7,30)$. Prescribing an ideal combination of AEDs for each patient could be a challenging process. A recent publication confirms that the trace element situation has been considerably changed in both conventional and newer AEDs (35).

The ABCB1-C3435T polymorphism is also likely to act as a risk factor for resistance to AEDs (36). The lack of sufficient data related to AEDs, poverty, cultural viewpoints, debates, poor health communications, and scarcity of skilled experts contribute to the management space (37). Adverse drug reactions such as hepatotoxicity, mitochondrial toxicity, hyperammonemic encephalopathy, hypersensitivity syndrome reactions, neurological toxicity, teratogenicity, metabolic and endocrine adverse events have been reported in relation to valproic acid $(7,38,39)$. Valproic acid also reduces alkaline phosphatase and hydroxyproline in lumbar spine (40). Skin reaction to at least one AED such as carbamazepine, phenytoin, lamotrigine or oxcarbazepine has been reported in 30 out of 300 patients; one of them developed Stevens-Johnson syndrome (34, 41-43).

AEDs-drug interactions are often associated with the cytochrome P450 (CYP450) enzymes. This group has more than 50 enzymes; six of them metabolize 90 percent of the drugs, among them the two most significant enzymes of CYP3A4 and CYP2D6. CYP450 can be inhibited or induced by drugs, resulting in clinically significant drugdrug interactions that can cause unanticipated adverse reactions or therapeutic failures.

Standard AEDs doses may cause side effects associated with eminent minimum concentration if a person metabolizes poorly or has a CYP450 enzyme inhibitor added to therapy. Adverse effects are more likely to occur in treatments with AEDs, because these drugs have a narrow therapeutic window and depend on enzyme CYP450 for their metabolism too. Potent inducers of CYP450 such as carbamazepine, phenytoin, and phenobarbital can cause clinically significant drug interaction by increasing enzyme synthesis (7, 44-48).

Finally, to prevent or decrease comorbid circumstances associated with epilepsy management, the alternative process should be based on considering the following guidelines to decrease AED-AED interaction: administration of preliminary dose, stabilizing drug level (minimum therapeutic concentration), increasing dosage, monitoring frequent and severe side effects, and predicting pharmacokinetics behavior.

\section{Acknowledgements}

Authors would like to express their special thanks to the Isfahan University of Medical Sciences. 


\section{Authors' Contributions}

All authors participated in data collection and manuscript preparation.

\section{Financial Disclosure}

The authors had no conflict of interest.

\section{Funding/Support}

Isfahan Neurosciences Research Center and Isfahan Deputy of Research supported this study (grant number 290296).

\section{References}

1. Chandra S, Kurwale N, Tripathi M, Banerjee J. Epileptogenic networks and drug-resistant epilepsy: Present and future perspectives of epilepsy research-Utility for the epileptologist and the epilepsy surgeon. Ann Indian Acad Neur. 2014;17(5):134.

2. Kalviainen R, Ansakorpi H, Flander S, Immonen A, Keranen T, Kivisto J, et al. [Update on Current Care Guideline: epilepsies (adult]. Duodecim. 2014;130(7):748-9.

3. Franchi C, Giussani G, Messina P, Montesano M, Romi S, Nobili A Validation of healthcare administrative data for the diagnosis of epilepsy. J Epidemiol Community Health. 2013;67(12).

4. Wiebe S. Definition of drug-resistant epilepsy: Is it evidence based? Epilepsia. 2013;54:9-12.

5. Weaver DF, Pohlmann-Eden B. Pharmacoresistant epilepsy: Unmet needs in solving the puzzle(s). Epilepsia. 2013;54:80-5.

6. Hao X, Goldberg D, Kelly K, Stephen L, Kwan P, Brodie MJ. Uncontrolled epilepsy is not necessarily the same as drug-resistant epilepsy: differences between populations with newly diagnosed epilepsy and chronic epilepsy. Epilepsy Behav. 2013;29(1):4-6.

7. Gregory JK, Broomall EM. Teaching Video NeuroImages: Reading epilepsy: A seizure in a thousand words (or less). Neurology. 2013;81(13):e100.

8. Cecen GS, Gulabi D, Oltulu I, Onay T. Generalized epileptic seizure in an adolescent idiopathic scoliosis (AIS) patient with syringomyelia after deformity correction surgery. Int J Surg Case Rep. 2013;4(8):740-3.

9. Lasoń W, Chlebicka M, Rejdak K. Research advances in basic mechanisms of seizures and antiepileptic drug action. Pharmacol Rep. 2013;65(4):787-801.

10. Tolou Ghamari Z. Antiepileptic Drugs (AEDs) Polypharmacy could lead to buried pharmacokinetic interactions due to CYP450. Drug Metab Lett . 2012;6(3):207-12.

11. Tolou-Ghamari Z, Najafi MR, Habibabadi JM, Zare M. Preliminarily Analysis of Carbamazepine (CBZ) C0 in Patients Visited Isfahan Epileptic Clinics. Int J Prev Med. 2013;4(Suppl 2):S343-6.

12. Tolou-Ghamari Z, Zare M, Habibabadi JM, Najafi MR. Antiepileptic drugs: a consideration of clinical and biochemical outcome in patients with epilepsy. Int J Prev Med. 2013;4(Suppl 2):S330-7.

13. Tolou-Ghamari Z, Zare M, Habibabadi JM, Najafi MR. A quick review of carbamazepine pharmacokinetics in epilepsy from 1953 to 2012. J Res Med Sci. 2013;18(Suppl 1):S81-5.

14. French JA, Faught E. Rational polytherapy. Epilepsia. 2009;50:63-8

15. Guberman A. Monotherapy or polytherapy for epilepsy? . Can J Neurol Sci.1998;25(4):S3-8.

16. Arroyo S, Perucca E. Translating monotherapy trials into clinical practice: a look into the abyss. Epilepsy Behav. 2003;4(5):457-63.

17. Glauser T, Ben-Menachem E, Bourgeois B, Cnaan A, Chadwick D, Guerreiro C, et al. ILAE Treatment Guidelines: Evidence-based Analysis of Antiepileptic Drug Efficacy and Effectiveness as Initial Monotherapy for Epileptic Seizures and Syndromes. Epilepsia. 2006;47(7):1094-120.

18. Macdonald RL, Kelly KM. Antiepileptic Drug Mechanisms of Action. Epilepsia.1995;36(s2):S2-S12.

19. Shank RP, Gardocki JF, Streeter AJ, Maryanoff BE. An Overview of the Preclinical Aspects of Topiramate: Pharmacology, Pharmacokinetics, and Mechanism of Action. Epilepsia. 2000;41(s1):3-9.

20. Luszczki JJ, Czuczwar M, Kis J, Krysa J, Pasztelan I, Swiader M, et al. Interactions of lamotrigine with topiramate and first-generation antiepileptic drugs in the maximal electroshock test in mice: an isobolographic analysis. Epilepsia. 2003;44(8):1003-13.

21. Contin M, Riva R, Albani F, Avoni P, Baruzzi A. Topiramate therapeutic monitoring in patients with epilepsy: effect of concomitant antiepileptic drugs. Ther Drug Monit. 2002;24(3):332-7.

22. Jenner P, Pratt JA, Marsden CD. Mechanism of action of clonazepam in myoclonus in relation to effects on GABA and 5-HT. Adv Neurol.1986;43:629-43.

23. Mallucci GR, Nowack A, Malarkey EB, Yao J, Bleckert A, Hill J, et al. Levetiracetam Reverses Synaptic Deficits Produced by Overexpression of SV2A. PLOS ONE. 2011;6(12).

24. Sankar R. GABAA Receptor Physiology and Its Relationship to the Mechanism of Action of the 1,5-Benzodiazepine Clobazam. CNS Drugs. 2012;26(3):229-44.

25. Tapalaga D, Suciu A, Schvartz M, Duca S. [The mechanism of action of phenobarbital on the metabolism of 131J-B.S.P. in cholestasis induced by ANIT in isolated and perfused rat liver (author's transl)]. Dtsch Z Verdau Stoffwechselkr. 1979;39(5):233-7.

26. Holder JL Jr, Wilfong AA. Zonisamide in the treatment of epilepsy. Expert Opin Pharmacother. 2011;12(16):2573-81.

27. Mula M, Trimble MR. Antiepileptic drug-induced cognitive adverse effects: potential mechanisms and contributing factors. CNS Drugs. 2009;23(2):121-37.

28. Onuma T. [Cognitive dysfunction and antiepileptic drugs]. Brain Nerve. 2011;63(4):379-83.

29. Semah F, Thomas P, Coulbaut S, Derambure P. Early add-on treatment vs alternative monotherapy in patients with partial epilepsy. Epileptic Disord. 2014.

30. Fröscher W, Rösche J. Fortschr Neurol Psychiatr. Combination Ther epilepsy. 2013;81(1):9-20.

31. Witt J, Elger CE, Helmstaedter C. Which drug-induced side effects would be tolerated in the prospect of seizure control? Epilepsy Behav. 2013;29(1):141-3.

32. Poduri A, Sheidley BR, Shostak S, Ottman R. Genetic testing in the epilepsies-developments and dilemmas. Nat Rev Neurol. 2014;10(5):293-9.

33. French JA, Gazzola DM. Antiepileptic drug treatment: new drugs and new strategies. Continuum (Minneap Minn). 2013;19(3 Epilepsy):643-55.

34. Yi Y, Lee JH, Suh ES. Toxic epidermal necrolysis induced by lamotrigine treatment in a child. Korean J Pedi. 2014;57(3):153.

35. Sarangi SC, Tripathi M, Kakkar AK, Gupta YK. Effect of antiepileptic therapy on trace elements status in Indian population in a tertiary care hospital from northern India: A cross sectional study. Epilepsy Res. 2014;108(5):917-27.

36. Li M, Tan J, Yang X, Su L, Xie J, Liang B, et al. The ABCB1-C3435T polymorphism likely acts as a risk factor for resistance to antiepileptic drugs. Epilepsy Res. 2014.

37. Satishchandra P, Santhosh N, Sinha S. Epilepsy: Indian perspective. Ann Indian Acad Neurol. 2014;17(5):3.

38. Nanau RM, Neuman MG. Adverse drug reactions induced by valproic acid. Clin Biochem. 2013;46(15):1323-38.

39. Pegg EJ, Zaman F. Sodium valproate-related hyperammonaemic encephalopathy. Case Rep. 2014;2014(apr10 1):bcr2014203899.

40. Anwar MI, Radhakrishna KV, Vohora D. Phenytoin and sodium valproate but not levetiracetam induce bone alterations in female mice. Can J Physiol Pharmacol. 2014.

41. Shingade PU, Wankhede V, Kataria PS, Sonone N. Rare case of phenytoin induced acute generalized exanthematous pustulosis with cerebellar syndrome. Indian J Dermatol. 2014;59(2):210.

42. Błaszczyk B, Szpringer M, Czuczwar SJ, Lasoń W. Single centre 20 year survey of antiepileptic drug-induced hypersensitivity reactions. Pharmacoll Rep. 2013;65(2):399-409.

43. Bourgeois BF. Initiating antiepileptic drug treatment and characteristics of drugs. Handb Clin Neurol. 2013;111:719-25.

44. Wilkinson GR. Drug metabolism and variability among patients in drug response. N Engl J Med. 2005;352(21):2211-21.

45. Slaughter RL, Edwards DJ. Recent advances: the cytochrome P450 enzymes. Ann Pharmacother.1995;29(6):619-24 
Tolou Ghamari Z et al.

46. Weinshilboum R. Inheritance and drug response. $N$ Engl J Med. 2003;348(6):529-37.

47. Phillips KA, Veenstra DL, Oren E, Lee JK, Sadee W. Potential role of pharmacogenomics in reducing adverse drug reactions: a sys- tematic review. JAMA. 2001;286(18):2270-9.

48. Bradford LD. CYP2D6 allele frequency in European Caucasians, Asians, Africans and their descendants. Pharmacogenomics. 2002;3(2):229-43. 